\title{
Gait asymmetries in unilateral symptomatic hip osteoarthritis and their association with radiographic severity and pain
}

\author{
Gary J Farkas ${ }^{1}$, Bryan R Schlink ${ }^{2}$, Louis F Fogg ${ }^{3}$, Kharma C Foucher ${ }^{4}$, Markus A \\ Wimmer $^{5,6}$, Najia Shakoor ${ }^{6}$ \\ ${ }^{1}$ Department of Physical Medicine and Rehabilitation, Pennsylvania State University College of \\ Medicine, Hershey, Pennsylvania, USA \\ 2Department of Biomedical Engineering, University of Florida, Gainesville, Florida, USA \\ ${ }^{3}$ College of Nursing, Rush University Medical Center, Chicago, Illinois, USA \\ ${ }^{4}$ Department of Kinesiology and Nutrition, University of Illinois at Chicago, Chicago, Illinois, USA \\ ${ }^{5}$ Department of Orthopedic Surgery, Rush University Medical Center, Chicago, Illinois, USA \\ ${ }^{6}$ Division of Rheumatology, Department of Internal Medicine, Rush University Medical Center, \\ Chicago, Illinois, USA
}

\section{Abstract}

Introduction: Little is known about the loading patterns in unilateral hip osteoarthritis (OA) and their relationship to radiographic severity and pain. We aimed to examine the loading patterns at the hips of those with unilateral symptomatic hip OA and identify associations between radiographic severity and pain with loading alterations.

Methods: 61 subjects with symptomatic unilateral hip OA underwent gait analyses and evaluation for radiographic severity (Kellgren-Lawrence [KL]-grade) and pain (visual analogue scale) at bilateral hips.

Results: Hip OA subjects had greater range of motion and higher hip flexion, adduction, internal and external rotation moments at the contralateral, asymptomatic hip compared to the ipsilateral hip $(p<0.05)$. Correlations were noted between increasing KL-grade and increasing asymmetry of contralateral to ipsilateral hip loading $(p<0.05)$. There were no relationships with pain and loading asymmetry.

Discussion: Unilateral symptomatic hip OA subjects demonstrate asymmetry in loading between the hips, with relatively greater loads at the contralateral hip. These loading asymmetries were directly related to the radiographic severity of symptomatic hip OA and not with pain.

Article reuse guidelines: sagepub.com/journals-permissions

Corresponding author: Gary J Farkas, Rehabilitation Research Laboratories, Department of Physical Medicine and Rehabilitation, Pennsylvania State University, College of Medicine, 500 University Drive, PO Box 850, Mail Code, R120, Hershey, PA 17033, USA. gfarkas@ pennstatehealth.psu.edu.

Declaration of conflicting interests

The author(s) declared no potential conflicts of interest with respect to the research, authorship, and/or publication of this article. 
Conclusion: Additional research is needed to determine the role of gait asymmetries in disease progression.

\section{Keywords}

Biomechanics; hip; loading; osteoarthritis; pain

\section{Introduction}

Osteoarthritis (OA) is the most common arthropathy worldwide and a major contributor to impaired quality of life and disability in older individuals. ${ }^{1-3}$ Biomechanics, including aberrant joint loading, have been known to contribute to cartilage degradation and play a significant role in lower extremity OA pathogenesis. ${ }^{4,5}$ The knees and hips are the most common joints affected by OA. ${ }^{6,7}$ Much of our information on aberrant joint loading in OA comes from studies of knees, ${ }^{8,9}$ and much less is known regarding alterations of loading patterns in OA of the hips.

Through biomechanical studies of lower extremity OA it has become apparent physical and mechanical alterations at one joint can have an effect at other joints. We have previously demonstrated that the contralateral knee of those with unilateral hip OA is more likely to develop end-stage OA than the ipsilateral knee, as well as exhibit higher peak moments, suggesting that joint mechanics affect the evolution of lower extremity OA. ${ }^{5,10}$ The most common pattern for OA to progress is to the cognate joint, which in the case for hip OA is the contralateral hip. ${ }^{10,11}$ Studies have documented altered biomechanics in hip OA and these alterations may play a role in the bilateral progression of the disease. ${ }^{12-15}$ However, what contributes to mechanical alterations, whether it is structural disease, pain, or a combination of these factors remains poorly understood. Increasing our understanding of these contributions may help to identify appropriate interventions to prevent disease progression.

This study had 2 aims focused on better understanding the mechanical alterations that occur in unilaterally symptomatic hip OA. The first aim was to evaluate alterations or asymmetries in loading between the hips in those that have unilateral symptomatic disease. The second aim was to explore how this asymmetry in loading was associated with radiographic severity and pain at the symptomatic hip. We hypothesised: (1) the contralateral hip of those with unilateral symptomatic hip OA would have higher dynamic joint loads relative to the symptomatic hip; and (2) radiographic severity and pain of the symptomatic hip would be independently related to degree of asymmetry in loading between the hip joints.

\section{Methods}

Subjects were part of a study approved by the Institutional Review Board of the host institution; the rights of the study participants were protected. Prior to participation all subjects completed informed consent. Data from this study cohort has previously been published. ${ }^{16}$ The current analyses used a slightly different sample from this cohort due to availability of complete data and subject criteria for analyses. Inclusion criteria included the presence of symptomatic OA of the hip as defined by the American College of 
Rheumatology's Clinical Criteria for Classification and Reporting of OA of the hip, ${ }^{17}$ and by the presence of at least $30 \mathrm{~mm}$ of pain (on a $100 \mathrm{~mm}$ scale) while walking (corresponding to question one of the visual analogue format of the hip-directed Western Ontario and McMaster Universities Arthritis Index [WOMAC] $).{ }^{18}$ Participants were excluded if they had evidence of radiographic $\mathrm{OA}$ of the contralateral hip or of either knee in excess of grade 3 according to the Kellgren-Lawrence (KL) grading scale. ${ }^{19}$ In addition, participants were excluded if they had greater than $20 \mathrm{~mm}$ of pain during walking at the contralateral hip or either knee. ${ }^{5,20}$ Other exclusion criteria included the inability to walk without assistance, presence of an inflammatory arthropathy, history of any lower extremity joint replacement, and history of trauma and/or surgery to either hip or knee within the preceding 6 months.

All subjects had AP radiographs performed of the pelvis. The KL scale was used to evaluate radiographic severity of both hips by a trained investigator. ${ }^{19}$ The WOMAC pain index was completed for each large lower extremity joint. ${ }^{18}$ The total pain score at the symptomatic hip was used in the analyses for this study (range 0-500 mm). Participants also underwent a clinical evaluation of the lower extremity joints. They denied pain at the contralateral hip (reported less than $20 \mathrm{~mm}$ out of $100 \mathrm{~mm}$ pain during normal walking on question one of the WOMAC) although they may have marked some pain on the complete WOMAC pain questionnaire. Nevertheless, they had asymmetric disease with acknowledgement of more pain at 1 hip over the contralateral side. For the purposes of this study and ease of discussion, we have labeled 1 hip "symptomatic" and the other hip the "contralateral hip" or "asymptomatic hip" with the acknowledgement that one is "relatively" more symptomatic than the other. Thus, based on the above criteria, the subjects enrolled in this study had symptomatic unilateral hip OA. These criteria have been used previously. 5,20

Gait analysis was performed at a single subject session according to previously published methods. ${ }^{21-23}$ A passive retroreflective marker set was placed bilaterally on lower extremity bony landmarks. Bony landmarks included the most anterior superior iliac spine, the centre of the greater trochanter, over the midpoint of the lateral joint line of the knee, the lateral most aspect of the lateral malleolus, the most lateral aspect of the base of the calcaneus, and the head of the 5th metatarsal. ${ }^{23} 4$ optoelectronic cameras (Qualisys, Gothenburg, Sweden) recorded the 3-dimensional (3D) positions of the reflective markers for each individual lower extremity by measuring the spatial position of the markers. Spatiotemporal parameters, including speed, stride, and cadence, as well as maximum hip extension, flexion, and range of motion (ROM), were calculated about the lower extremity as previously described. ${ }^{23,24}$ These calculations were based on the 3D positions of the markers. The 3D positions of the joint centers were estimated based on the marker location and anthropometric measurements. The joint center for the knee joint was determined to be the midpoint between the medial and lateral tibial and femoral condyles; the midpoint between the medial and lateral malleoli was determined to be the joint centre of the ankle joint. The joint centre of the hip was determined to be $2.5 \mathrm{~cm}$ distal from the midpoint between the anterior superior iliac spine and the pubic tubercle. ${ }^{25}$

A multicomponent floor-embedded force plate (Bertec, Columbus, Ohio), with a sampling frequency of $120 \mathrm{~Hz}$, measured the location and magnitude of the ground reaction force (GRF). An inverse dynamics approach was used to calculate external joint moments which 
were normalised to percent body weight times height (\%BWH). ${ }^{23,24,26}$ Briefly, these calculations were based on the 3D position of the passive reflective markers, anthropometry measurements used to localise the joint centres relative to the marker positions, and the magnitude and location of the GRF. Newton's second law explains that peak external moments that act on a joint are equal and opposite to the net internal moments produced by joint contact forces, muscles, and soft tissues at the joint. All peak external moments were normalised to percent body weight times height $(\% \mathrm{BW} * \mathrm{H}) .{ }^{26}$ Gait data was processed using the MotionMonitor (Innovative Sports Training Inc., Chicago, Illinois). Subjects were instructed to walk at a normal self-selected walking speed across a 6-metre walkway completing a total of 5 runs per limb while wearing their own shoes. 2 to 3 practice trials were completed prior to performing the walking runs to limit speed variability and familiarise the subjects with the walking runs. Mean gait data for the 5 walking runs of the asymptomatic and symptomatic limbs were compared. The individual analysing the runs was blinded to the affected side.

Asymmetry calculations were measured according to previously published methods. ${ }^{27-30}$ Briefly, extent of asymmetry in loading was evaluated as a ratio of loading of the symptomatic hip to the asymptomatic hip (i.e. symptomatic:asymptomatic hip ratio). The same ratio was calculated for ROM. A value of 1 denotes complete symmetry between the hips, whereas a value of $<1$ or $>1$ denotes relative asymmetry. A ratio $<1$ would represent asymmetry with lower loads or less ROM at the symptomatic hip, whereas a value $>1$ would represent asymmetry with higher loads and more ROM at the symptomatic hip.

All variables were evaluated for normality. A chi-squared test was used to evaluate the distribution of KL grades between the symptomatic and asymptomatic hips. 2-tailed paired sample $t$-test were used to examine differences in gait parameters between the hips. Spearman rho correlations were used to determine if relationships were present between pain and KL-grade of the symptomatic hip and asymmetries in gait variables. For the correlations, a Bonferroni correction was used with a $p<0.005$ considered statistically significant. All data were analysed using SPSS statistical software (SPSS Inc., IBM, Armonk, NY).

\section{Results}

61 participants with symptomatic unilateral hip OA qualified to undergo analysis. There were 25 males and 36 females with a mean (standard deviation [SD]) age and body mass index (BMI) of 62 (11) years and $28(5) \mathrm{kg} / \mathrm{m}^{2}$, respectively. For KL grade at the clinically symptomatic hip, the majority of subjects $(n=43)$ had a KL grade of $\geq 3$, whereas at the asymptomatic hip the majority of subjects $(n=46)$ had a KL grade of $\mathcal{s}$ (Table 1$)$. The distribution of KL grades between the symptomatic and asymptomatic hips were significantly different $\left(\chi^{2}=40.37, p<0.001\right.$; Table 1$)$. The symptomatic hip had significantly higher WOMAC pain values compared to the asymptomatic hip $(p<0.0001$; Table 1).

When examining spatiotemporal parameters between the symptomatic and asymptomatic hip no differences were observed between speed, stride, and cadence ( $p>0.05$; Table 2 ).

Hip Int. Author manuscript; available in PMC 2020 July 03. 
Significantly greater overall hip ROM in the sagittal plane as well as maximum hip extension and maximum hip flexion were found at the asymptomatic hip compared to the symptomatic hip ( $p \leq 0.005$; Table 2 ). The asymptomatic hip also had significantly higher peak external moments including the peak hip flexion, adduction, internal and external rotation moments compared to the symptomatic hip $(p \leq 0.018)$. No significant differences were observed between the joints for the peak external hip extension and abduction moments $(p>0.05$; Table 2).

Correlations between radiographic severity and pain with gait asymmetries are presented in Table 3. Results showed that as KL-grade increased at the symptomatic hip, the asymmetry loading ratio decreased (i.e. the contralateral, asymptomatic hip had relatively higher dynamic loads compared to the symptomatic hip). This was the case for the hip flexion, adduction, internal rotation moments. With regards to hip ROM, with increasing KL-grade, the contralateral, asymptomatic hip had greater overall ROM in the sagittal plane relative to the symptomatic hip (Table 3). There were no significant relationships observed between asymmetries and pain in loading or ROM on at the hips (Table 3).

\section{Discussion}

This study focused on evaluating gait asymmetries between the hips in unilaterally symptomatic hip OA and the relationship of these gait patterns to radiographic and pain severity of the affected hip. Subjects were considered to have clinically symptomatic unilateral hip OA due to the presence of both radiographic changes and localised hip pain, even in the presence of some radiographic degeneration in the contralateral hip however with no or minimal report of pain. ${ }^{17}$ The main findings from this study suggest: (1) that there is unloading of the symptomatic hip with a relative overloading of the contralateral, asymptomatic hip; and (2) this loading asymmetry is associated with OA radiographic severity but not with pain at the symptomatic hip.

Our findings of less ROM at the symptomatic joint and relative unloading of the symptomatic joint are consistent with previous studies. ${ }^{12,31}$ Moreover, this study suggests that pain had little or no association to gait asymmetries, while radiographic changes had significant associations as in line with previous investigations. ${ }^{32-34}$ Tateuchi et al. ${ }^{32}$ reported associations between minimal joint space narrowing and sagittal plane loading at the hip. Foucher et al. ${ }^{34}$ demonstrated that with increasing KL-grade subjects exhibited a greater degree of gait abnormalities known as motion discontinuity. The current study provides further support that changes in joint morphology rather than symptoms may provide a better predictor of variant gait in those with hip OA.

There are several clinical implications for this study's findings. We theorise that subjects reduce joint excursion and loads at the symptomatic joint and relatively increase at the contralateral side as a compensatory mechanism. It may be that these gait alterations are purely related to structural changes. This is consistent with the theories regarding the pathophysiology of femoroacetabular impingement where the pathomorphology of the hip joint is thought to cause a change in the moment arm about the hip, ultimately contributing to the gait alterations and early OA. ${ }^{35}$ We have also previously demonstrated that limb 
loading asymmetries in unilateral hip OA are associated with neuromuscular alterations including quadriceps strength and proprioception at the knees. ${ }^{16}$ Radiographic severity could serve as a biomarker of alterations of neuromuscular factors at the joint that is potentially modifiable with rehabilitation and/or surgical interventions. Thus, rehabilitative professionals may be able to intervene during early stages of the disease with strategies to normalise gait patterns and potentially delay the progression of OA to subsequent joints. ${ }^{10}$

\section{Limitations}

This study is not without limitations. First, the cross-sectional design of this study did not establish causality. Second, hip ROM was only analysed in the sagittal plane as the greatest range of motion during gait occurs in this plane. Furthermore, our marker system did not allow for reliable evaluation of pelvic and/or trunk biomechanics, which may be an interesting aspect for future, studies to evaluate. We did not have data available on duration of hip OA symptoms. We used the contralateral, asymptomatic hip and not healthy controls for our comparisons since we believe this provides a better model to study overall multiarticular OA pathophysiology. Although our subjects may have had some radiographic OA at both hips, 1 was considered clinically more symptomatic as evident by greater radiographic severity and WOMAC pain scores. Radiographic OA is not uncommon in this age group thus these subjects might be more representative of a normal population than if radiographic OA of the contralateral hip had been completely excluded. Therefore, this study used a more clinically applicable definition of symptomatic unilateral hip OA. Further, recent research shows that in knee OA, asymptomatic individuals with KL-grade 2 knees are biomechanically homologous with those who have KL-grade 0 or 1 at the knee, while those who have KL-grade 2 knees paired with pain symptoms have significantly higher peak adduction moments. ${ }^{20,36}$ This suggests that clinically symptomatic OA is biomechanically distinct from isolated radiographic disease. ${ }^{20,36}$ Lastly, although we did not find consistent associations with pain, perhaps the relationships between pain and loading alterations are present at certain time points, such as early or at the end-points of the disease which this study was not able to elucidate.

\section{Conclusion}

In summary, unilateral hip OA subjects presented with loading asymmetries between the hips which were directly related to the radiographic severity of hip OA but not to symptoms of pain. Subjects demonstrated reduced joint range of motion and loads at the symptomatic hip and relative overloading at the contralateral hip joint. To what extent the observed asymmetries may be improved through rehabilitative intervention warrants further investigation.

\section{Acknowledgments}

Funding

The author(s) disclosed receipt of the following financial support for the research, authorship, and/or publication of this article: The authors would like to thank the National Institute of Arthritis and Musculoskeletal and Skin Diseases (K23AR049748), the National Institute on Aging (AG024891), and the Schweppe Foundation for their support of this study. The sponsors of this study did not partake in the design of the study; the collection, analysis, 
or the interpretation of the data; the writing of the manuscript, or the decision to submit the manuscript for publication.

\section{References}

1. Centers for Disease Control and Prevention. Prevalence of doctor-diagnosed arthritis and arthritisattributable activity limitation--United States, 2010-2012. MMWR Morb Mortal Wkly Rep 2013; 62: 869-873. [PubMed: 24196662]

2. Hootman JM and Helmick CG. Projections of US prevalence of arthritis and associated activity limitations. Arthritis Rheum 2006; 54: 226-229. [PubMed: 16385518]

3. Zhang Y and Jordan JM. Epidemiology of osteoarthritis. Clin Geriatr Med 2010; 26: 355-369. [PubMed: 20699159]

4. Jr Bowman KF, Fox J and Sekiya JK. A clinically relevant review of hip biomechanics. Arthrosc J Arthrosc Relat Surg 2010; 26: 1118-1129.

5. Shakoor N, Hurwitz DE, Block JA, et al. Asymmetric knee loading in advanced unilateral hip osteoarthritis. Arthritis Rheum 2003; 48: 1556-1561. [PubMed: 12794823]

6. Hunter DJ and Felson DT. Osteoarthritis. BMJ 2006; 332: 639-642. [PubMed: 16543327]

7. Quintana JM, Arostegui I, Escobar A, et al. Prevalence of knee and hip osteoarthritis and the appropriateness of joint replacement in an older population. Arch Intern Med 2008; 168: 15761584. [PubMed: 18663171]

8. Kaufman KR, Hughes C, Morrey BF, et al. Gait characteristics of patients with knee osteoarthritis. J Biomech 2001; 34: 907-915. [PubMed: 11410174]

9. Maly MR. Abnormal and cumulative loading in knee osteoarthritis. Curr Opin Rheumatol 2008; 20 : 547-552. [PubMed: 18698176]

10. Shakoor N, Block JA, Shott S, et al. Nonrandom evolution of end-stage osteoarthritis of the lower limbs. Arthritis Rheum 2002; 46: 3185-3189. [PubMed: 12483722]

11. Dougados M, Gueguen A, Nguyen M, et al. Radiological progression of hip osteoarthritis: definition, risk factors and correlations with clinical status. Ann Rheum Dis 1996; 55: 356-362. [PubMed: 8694574]

12. Ornetti P, Laroche D, Morisset C, et al. Three-dimensional kinematics of the lower limbs in hip osteoarthritis during walking. J Back Musculoskelet Rehabil 2011; 24: 201-208. [PubMed: 22142708]

13. Ometti P, Maillefert J-F, Laroche D, et al. Gait analysis as a quantifiable outcome measure in hip or knee osteoarthritis: a systematic review. Joint Bone Spine 2010; 77: 421-425. [PubMed: 20471899]

14. Eitzen I, Fernandes L, Kallerud H, et al. Gait characteristics, symptoms, and function in persons with hip osteoarthritis: a longitudinal study with 6 to 7 years of follow-up. J Orthop Sports Phys Ther 2015; 45: 539-549. [PubMed: 25996360]

15. Foucher KC and Wimmer MA. Contralateral hip and knee gait biomechanics are unchanged by total hip replacement for unilateral hip osteoarthritis. Gait Posture 2012; 35: 61-65. [PubMed: 21903396]

16. Shakoor N, Foucher KC, Wimmer MA, et al. Asymmetries and relationships between dynamic loading, muscle strength, and proprioceptive acuity at the knees in symptomatic unilateral hip osteoarthritis. Arthritis Res Ther 2014; 16: 455. [PubMed: 25496937]

17. Altman R, Alarcon G, Appelrouth D, et al. The American College of Rheumatology criteria for the classification and reporting of osteoarthritis of the hip. Arthritis Rheum 1991; 34: 505-514. [PubMed: 2025304]

18. Bellamy N, Buchanan WW, Goldsmith $\mathrm{CH}$, et al. Validation study of WOMAC: a health status instrument for measuring clinically important patient relevant outcomes to antirheumatic drug therapy in patients with osteoarthritis of the hip or knee. J Rheumatol 1988; 15: 1833-1840. [PubMed: 3068365]

19. Kellgren JH and Lawrence JS. Radiological assessment of osteo-arthrosis. Ann Rheum Dis 1957; 16: 494-502. [PubMed: 13498604] 
20. Shakoor N, Dua A, Thorp L, et al. Asymmetric loading and bone mineral density at the asymptomatic knees of subjects with unilateral hip osteoarthritis. Arthritis Rheum 2011; 63: 38533858. [PubMed: 22127702]

21. Andriacchi TP, Natarajan R and Hurwitz D. Musculoskeletal dynamic locomotion and clinical applications. Philadelphia: Lippincott, 2005.

22. Andriacchi TP. Dynamics of pathological motion: applied to the anterior cruciate deficient knee. J Biomech 1990; 23(Suppl. 1): 99-105. [PubMed: 2081748]

23. Prodromos CC, Andriacchi TP and Galante JO. A relationship between gait and clinical changes following high tibial osteotomy. J Bone Joint Surg Am 1985; 67: 1188-1194. [PubMed: 4055843]

24. Andriacchi TP, Galante JO and Fermier RW. The influence of total knee-replacement design on walking and stair-climbing. J Bone Joint Surg Am 1982; 64: 1328-1335. [PubMed: 7142241]

25. Andriacchi TP and Strickland AB. Gait analysis as a tool to assess joint kinetics In: Berme N, Engin AE, da Silva KMC (eds) Biomechanics of normal and pathological human articulating joints. Dordrecht: Martiuns Nijhof, 1985, pp. 83-102.

26. Moisio KC, Sumner DR, Shott $S$, et al. Normalization of joint moments during gait: a comparison of two techniques. J Biomech 2003; 36: 599-603. [PubMed: 12600350]

27. Christiansen CL and Stevens-Lapsley JE. Weight-bearing asymmetry in relation to measures of impairment and functional mobility for people with knee osteoarthritis. Arch Phys Med Rehabil 2010; 91: 1524-1528. [PubMed: 20875509]

28. Kutilek P, Viteckova S, Svoboda Z, et al. Kinematic quantification of gait asymmetry in patients with peroneal nerve palsy based on bilateral cyclograms. J Musculoskelet Neuronal Interact 2013; 13: 244-250. [PubMed: 23728111]

29. Seliktar R and Mizrahi J. Some gait characteristics of below-knee amputees and their reflection on the ground reaction forces. Eng Med 1986; 15: 27-34. [PubMed: 3699235]

30. Crenshaw SJ and Richards JG. A method for analyzing joint symmetry and normalcy, with an application to analyzing gait. Gait Posture 2006; 24: 515-521. [PubMed: 16427288]

31. Hurwitz DE, Hulet CH, Andriacchi TP, et al. Gait compensations in patients with osteoarthritis of the hip and their relationship to pain and passive hip motion. J Orthop Res 1997; 15: 629-635. [PubMed: 9379275]

32. Tateuchi H, Koyama Y, Tsukagoshi R, et al. Associations of radiographic degeneration and pain with daily cumulative hip loading in patients with secondary hip osteoarthritis. J Orthop Res Off Publ Orthop Res Soc. Epub ahead of print 4 March 2016 DOI: 10.1002/jor.23223.

33. Eitzen I, Fernandes L, Nordsletten L, et al. Sagittal plane gait characteristics in hip osteoarthritis patients with mild to moderate symptoms compared to healthy controls: a cross-sectional study. BMC Musculoskelet Disord 2012; 13: 258. [PubMed: 23256709]

34. Foucher KC, Schlink BR, Shakoor N, et al. Sagittal plane hip motion reversals during walking are associated with disease severity and poorer function in subjects with hip osteoarthritis. J Biomech 2012; 45: 1360-1365. [PubMed: 22498313]

35. Farkas GJ, Cvetanovich GL, Rajan KB, et al. Impact of Femoroacetabular Impingement Morphology on Gait Assessment in Symptomatic Patients. Sports Health 2015; 7: 429-436. [PubMed: 26502419]

36. Thorp LE, Sumner DR, Wimmer MA, et al. Relationship between pain and medial knee joint loading in mild radiographic knee osteoarthritis. Arthritis Care Res 2007; 57: 1254-1260. 
Table 1.

Radiographic severity and pain of subjects.

\begin{tabular}{lcccccl}
\hline Hip & \multicolumn{1}{c}{ KL Grade $^{*}(n)$} & & $\begin{array}{l}\text { WOMAC Pain } \\
\text { Mean (SD) }\end{array}$ \\
\cline { 2 - 6 } & KL 1 & KL 2 & KL 3 & KL 4 & \\
\hline Symptomatic $^{\S}$ & 3 & 15 & 20 & 23 & $\begin{array}{l}179(120) \\
\text { (range 22-458) }\end{array}$ \\
Asymptomatic & 15 & 34 & 12 & 0 & $\begin{array}{l}27(36) \\
\text { (range 0.00-157.0) }\end{array}$ \\
\hline
\end{tabular}

Kellgren-Lawrence grading scale; significantly different distribution between the KL-grade of symptomatic and asymptomatic hips $\left(\otimes^{2}=40.37, p\right.$ $<0.001)$.

${ }^{\dagger}$ Western Ontario and McMaster Universities Arthritis pain scale in millimetres (range 0-500 mm).

$\mathrm{SD}$, standard deviation. 


\section{롤 \\ 올}

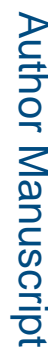

$\frac{1}{2}$

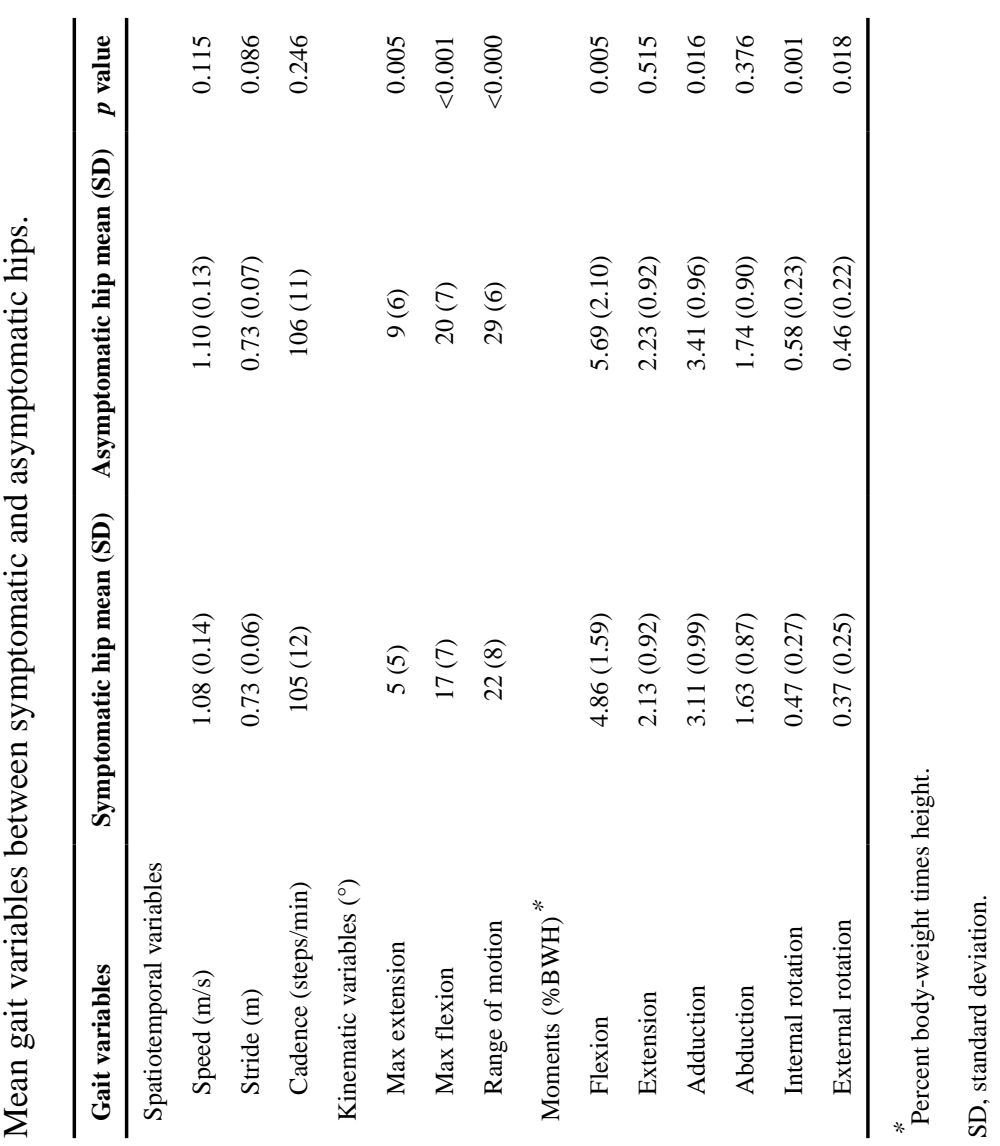

Hip Int. Author manuscript; available in PMC 2020 July 03. 


\section{롤 \\ 골}

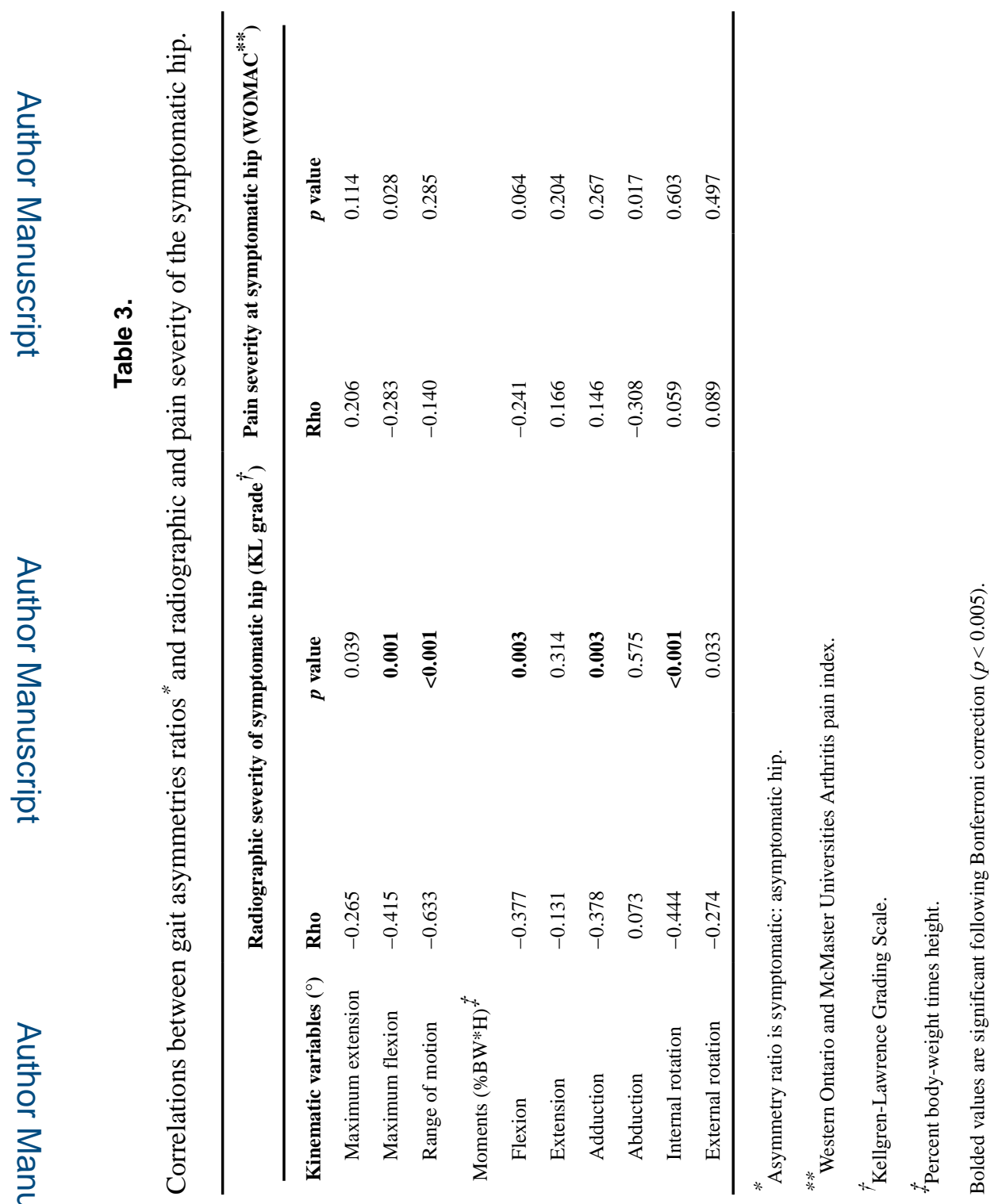

Hip Int. Author manuscript; available in PMC 2020 July 03 